Slavica

bruxellensia

\section{Slavica bruxellensia}

Revue polyphonique de littérature, culture et histoire slaves

6 | 2010

Linguistique russe

\title{
Entretien avec Aleksandr A. Puškin \& Maria A. Puškinova
}

\section{Eric Metz et Katia Vandenborre}

\section{OpenEdition \\ Journals}

Édition électronique

URL : http://journals.openedition.org/slavica/352

DOI : 10.4000/slavica.352

ISSN : 2034-6395

\section{Éditeur}

Université libre de Bruxelles - ULB

\section{Édition imprimée}

Pagination : 41-47

ISSN : 2031-7654

Référence électronique

Eric Metz et Katia Vandenborre, «Entretien avec Aleksandr A. Puškin \& Maria A. Puškinova », Slavica bruxellensia [En ligne], 6 | 2010, mis en ligne le 15 juin 2010, consulté le 21 avril 2019. URL : http:// journals.openedition.org/slavica/352 ; DOI : 10.4000/slavica.352

Ce document a été généré automatiquement le 21 avril 2019

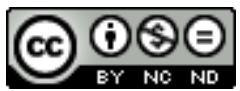

Les contenus de Slavica bruxellensia sont mis à disposition selon les termes de la Licence Creative Commons Attribution - Pas d'Utilisation Commerciale - Pas de Modification 3.0 France. 


\title{
Entretien avec Aleksandr A. Puškin \& Maria A. Puškinova
}

\author{
Eric Metz et Katia Vandenborre
}

\section{Présentation}

1 Aleksandr Puškin, le «soleil de la poésie russe ", n’a jamais mis pied dans nos régions, mais c'est bien en Belgique que vivent ses deux descendants les plus directs: Aleksandr Aleksandrovič Puškin et son épouse Maria Aleksandrovna, lesquels s'efforcent de mieux faire connaître le nom de Puškin, notamment par le biais de la Fondation Internationale A. S. Pouchkine. Les époux Puškin ont eu la gentillesse de nous accueillir dans leur appartement bruxellois et de nous guider à travers l'histoire fascinante de leur famille ainsi que de l'émigration russe qui a suivi la Révolution d'Octobre.

2 Au nom de la rédaction de «SLAVICA BRUXELLENSIA » nous les remercions pour l'entretien qu'ils nous ont accordé.

\section{Entretien}

Katia Vandenborre : Pourriez-vous nous esquisser l'arbre généalogique de votre famille?

Aleksandr Puškin: Le nom " Puškin » est un nom russe très ancien, il remonte aux temps des Vikings, bien qu'à l'époque on n'utilisait que des surnoms et non des noms de famille. Au XIV ${ }^{e}$ siècle, apparut le surnom «Puška », qui signifie «canon » en russe. Pratiquement cent ans après, on retrouve officiellement le nom de Puškin à Nižnij Novgorod, où celui qui portait ce nom possédait des terres. La famille Puškin recevra dans ses armoiries une couronne princière sur de l'hermine car neuf Puškin se prononcèrent pour l'avènement des Romanov au trône en 1613. Protecteurs de la couronne, les Puškin occupèrent des postes très importants : ambassadeurs, conseillers, sénéchaux, etc. Le père d'Aleksandr Sergeevič était officier dans l'armée russe, sa mère était d'origine abyssine. En effet, elle était la petite-fille d'Abraham Hannibal, un prince 
éthiopien qui, fait prisonnier par les Turcs et racheté par l'ambassadeur de Russie, avait été offert par ce dernier à Pierre le Grand. Le trouvant très intelligent, le tsar prit l'initiative de le former, notamment en France. À son retour en Russie, Hannibal occupa des postes clés, entre autres dans l'armée impériale, où il devint général.

Eric Metz : Qu'en est-il d'Aleksandr Sergeevič lui-même?

A. P. : Marchant dans les traces de son aïeul, il étudia au Lycée Impérial Alexandre, nouvellement fondé en 1811 pour former l'élite. Le français fut sa première langue et celle de ses premiers poèmes. Le russe, qu'il apprit vers l'âge de sept ans grâce à sa grand-mère et sa nounou prit ensuite le relais dans ses œuvres. Les écrits de Puškin lui causèrent à plusieurs reprises des soucis avec la censure, exigeant parfois l'intervention de ses amis du Lycée, parmi lesquels Nikolaj Žukovskij.

Pour reprendre la généalogie, le mariage de Puškin avec NataLja Gončarova ne reçut pas immédiatement l'aval de la famille de celle-ci en raison de la vie mouvementée du poète et de la grande différence d'âge qu'il y avait entre eux : elle avait seize ans et lui trente. Ils durent attendre deux ans. Ensemble, ils eurent quatre enfants: Maria, NataLja, Grigorij et Aleksandr. Ce dernier, qui devint général, est notre trisaïeul à tous les deux. Il eut treize enfants, parmi lesquels il y avait, du premier mariage, l'arrièregrand-mère de mon épouse et, du deuxième mariage, mon grand-père. Il y a donc un décalage de génération : je suis au cinquième niveau et ma femme au sixième.

K. V. : Comment ces deux branches de la famille se sont-elles rencontrées ?

A. P. :Nos grands-parents se sont rencontrés en Turquie. Après la révolution, les Puškin sont d'abord partis dans le sud de la Russie, où ils avaient une propriété. Puis, comme la situation ne se calmait pas, ils ont traversé la Mer Noire et sont arrivés en Turquie. Nos grands-parents vécurent au même endroit. Ensuite, en raison des difficultés liées à la langue, ils décidèrent de quitter le pays. Les grands-parents de ma femme ont choisi Paris. Les miens, par contre, ont rejoint la Yougoslavie car non seulement ma grandmère y avait une sœur, mais c'était aussi un royaume, où l'on parlait en outre une langue slave. Néanmoins, ils ne s'y plurent pas. Mon grand-père, qui travaillait alors pour la Croix Rouge, apprit qu'en Belgique le cardinal Mercier faisait beaucoup pour les émigrés russes. Il lui écrivit une lettre expliquant la situation dans laquelle se trouvait sa famille. Le cardinal Mercier y réagit positivement, les invitant à venir en Belgique. Ils arrivèrent en 1923. Mon père fit des études à Saint-André de Bruges, puis à Saint-Louis à Bruxelles et s'établit ainsi en Belgique, tout en continuant d'entretenir une relation épistolaire avec la mère de mon épouse. C'est de cette manière qu'en 1949 les deux familles partirent ensemble en vacances à la Côte d'Azur, où Maria et moi nous nous rencontrâmes pour la première fois. Nous ne nous revirent plus avant 1960, où le même topo se répéta. Elle avait alors seize ans, moi dix-huit. Nous commençâmes à flirter. Cela dura neuf ans car j'étudiais à Bruxelles et Maria à Paris. Enfin, après les études et le service militaire, nous nous marièrent en 1970. Nous n'eûmes malheureusement pas d'enfant... C'est pourquoi nous créâmes une Fondation en 1999.

E. M. : À ce propos, comment en êtes-vous arrivés à créer cette Fondation?

Maria Puškina: Ne pouvant avoir d'enfants et étant les derniers du nom, nous nous disions que nous devions laisser quelque chose après nous à la mémoire de Puškin. Ainsi, en 1999, à l'occasion du bicentenaire de la naissance de Puškin, nous avons fondé cette Fondation Internationale A.S. Pouchkine. Elle a d'une part pour objet les échanges culturels, littéraires et artistiques, essentiellement entre la Russie et la 
Belgique. D'autre part, ces activités ont pour objectif de financer son volet caritatif étant donné que les fonds récoltés sont destinés au département de pédiatrie d'un hôpital d'oncologie à Saint-Pétersbourg. Ainsi, chaque année, nous achetons des appareils pour eux. Pour ce faire, nous organisons une à deux manifestations par an.

E. M. : De quelle nature sont ces manifestations?

M. P. :Littéraire, musicale, cinématographique, etc. Nous tentons de diversifier nos activités. Nous avons par exemple invité le jazzman Alexandre Cavaliere et Johan Schmidt, le lauréat du Concours Reine Elisabeth pour un concert de Pëtr ILǐč Čajkovskij, nous avons projeté Pouchkine, le dernier duel de Natal'ja Bondarčuk (2006), nous avons programmé "Une nuit à Saint-Pétersbourg avec Pouchkine» au Théâtre Poème. Ce spectacle, qui illustre la vie de Puškin au travers de ses œuvres, eut un succès tel qu'il fut présenté au Café Littéraire à Saint-Pétersbourg, le café où Puškin prit sa dernière collation avant son duel, ainsi qu'à l'ambassade de Belgique à Moscou.

K. V. : Tout en privilégiant la personne de Puškin, vos activités embrassent donc toute la culture russe, que vous vous appliquez à transmettre à un public belge...

M. P. : Oui, nous visons tant le public wallon que flamand ou russe. Il n'est pas question de barrières.

E. M. : Vos activités sont-elles soutenues par la Russie?

A. P. :Très peu. Ils nous aident si nous avons besoin d'eux, essentiellement par le biais de l'ambassade.

E. M. : Nous avons remarqué sur votre site Internet que vous aviez le projet de publier un recueil de poésie d'un auteur russe en émigration. Pouvez-vous nous dire comment évolue ce projet?

M. P. :Nous n'avons malheureusement pas encore pu le réaliser par manque de fonds. Le projet est de constituer un recueil bilingue, c'est-à-dire en français et en néerlandais, voire trilingue, avec l'original russe. Mais nous n'avons pas encore pu trouver de sponsor.

E. M. : Avez-vous des contacts avec le monde littéraire russe en Belgique?

M. P. :Très peu, car ce monde est très réduit dans les faits.

K. V. : Les émigrés russes sont-ils très actifs sur le plan culturel en Belgique?

A. P. :Oui. Il y a notamment des pièces qui sont montées en russe chaque année, autrement dit pour un public russophone. Cela relève d'une volonté qu'ont les émigrés de garder un contact avec leurs racines. Nombreux sont ceux qui perdent leur langue maternelle.

E. M. : Vous êtes issus de l'émigration blanche. Cela constitue-t-il toujours une identité à I'heure actuelle?

M. P. : Oui. D'autres émigrations ont suivis après et nous ne nous identifions pas à elles.

A. P.:De plus, nous gardons toujours cette idée de la Russie ancienne bien que nous ayons évolué. Nous comprenons que la Russie devait changer, mais, finalement, au vu de ce qu'a accompli Stalin, rien n'a changé. La mémoire de l'émigration blanche et de ceux qui ont lutté contre le communisme persiste en Belgique. D'ailleurs, très récemment, une plaque a été apposée sur la maison du général Pëtr Nikolaevič Vrangel, adversaire du bolchévisme, qui vivait avenue Bel-Air à Uccle. Il y a donc toujours l'esprit de l'ancienne Russie... 


\section{K. V. : L'Association de la Noblesse Russe y a sans doute son rôle à jouer...}

A. P. :J'en suis le président. L'Association réunit une vingtaine de familles russes, ce qui fait environ cent cinquante membres. Nous nous réunissons chaque année lors d'un souper et nous organisons deux ou trois conférences par an sur un sujet russe. Dernièrement, le prince Khoroskoj a fait une conférence sur le thème de «Moscou, troisième Rome ». Encore avant, un moine de l'abbaye bénédictine de Chevetogne a expliqué pourquoi le rite byzantin y côtoie le rite romain. Vadim Lukov, l'ancien ambassadeur de Belgique en Russie, a parlé quant à lui des rapports politiques entre la Belgique et la Russie.

E. M. : L'idée communément admise aujourd'hui est que le communisme n'existe plus. Mais il n'y a pas si longtemps de cela, il en était autrement. L'Europe, en ce compris la Belgique, avait beaucoup de sympathie pour cette idéologie. Je pense à la période de la Deuxième Guerre mondiale et après celle-ci. Quels sont les souvenirs que vous en avez ou que vous ont racontés vos parents sur cette époque? Avaient-ils certaines difficultés à s'exprimer en tant qu'anti-communistes?

A. P. :Non. Nous avons tout perdu et avons toujours considéré que le communisme n'avait rien apporté de bon. Le peuple était malheureux. À moins de suivre les textes fondateurs à la lettre, c'est une idéologie qui n'est pas applicable.

M. P. :D'ailleurs, nous nous demandons comment un parti communiste peut encore exister au XXI ${ }^{\mathrm{e}}$ siècle, avec tout ce qui s'est passé.

\section{K. V. : Pour revenir à vos parents, comment s'est passée leur intégration ?}

M. P. :il n'y a pas eu de problème puisqu'ils parlaient le français et étaient chrétiens. Ma grand-mère ne travaillait pas et mon grand-père, qui était juge de paix, s'est mis à réparer des téléphones...

A. P. :Ils ont tout perdu et ont tout reconstruit. Mon grand-père était juge. Arrivé à l'âge de quarante ans, il était trop tard pour faire carrière, alors il s'est lancé dans l'héraldique. Après des études commerciales, mon père, lui, a travaillé dans le journal « LES SPORTS ». Ce qui est très amusant, c'est que d'après Wim Coudenys ${ }^{1}$, qui a fait des recherches dans des archives, mon père a été baptisé catholique à Saint-André probablement sans le savoir puisqu'il s'est marié à l'église orthodoxe, m'a baptisé selon le rite orthodoxe...

E. M. : Wim Coudenys a publié un ouvrage qui a fait parler de lui puisqu'il y présentait la thèse selon laquelle des émigrés russes auraient collaboré avec l'Allemagne pendant la Deuxième Guerre mondiale². Pourriez-vous commenter cette thèse?

A. P. :Avant la guerre déjà, tous les Russes de l'âge de mon père, qui avaient alors entre vingt-cinq et trente ans, espéraient tous rentrer en Russie. Ils ont alors fondé une école militaire pour les Russes, reconnue par l'État belge. Ils avaient des armes, ils s'entraînaient sur l'actuel site de la RTBF pour se préparer à combattre. Ils n'ont pas pris part au combat dès le déclenchement de la guerre. C'est seulement quand l'armée allemande est entrée en Russie qu'ils ont vu un moyen de vaincre le régime communiste en entrant dans l'armée allemande. Tous les Russes n'ont pas collaboré. Certains étaient dans la résistance belge.

M. P. :Je crois que l'erreur de jugement de Wim Coudenys provient aussi de l'influence de Vladimir Ronin ${ }^{3}$ et son incompréhensible discréditation de la communauté russe. Lui-même avait présenté l'idée selon laquelle les Russes en Afrique avaient été des esclavagistes, ce qui a suscité un tollé lors d'une conférence sur l'émigration russe. 
E. M. : Est-ce que les Puškin vivent un peu partout dans le monde?

A. P. :Oui. L'année passée, Jurij Lužkov, le maire de Moscou, a organisé une réunion de tous les descendants de Puškin. Tous ne sont pas venus : nous n'étions que quatre-vingt environ sur les deux cent trente-six répartis dans le monde entier. Néanmoins, je suis le dernier à porter le nom de Puškin, les autres étant descendants par les femmes.

K. V. : Êtes-vous en contact avec eux?

A. P. :Nous le sommes avec certains, mais essentiellement en Europe. En Angleterre, ils sont liés à la famille royale, nous n'avons pas de contact avec eux.

E. M. : Quel est le lien entre Puškin et la Belgique?

A. P. :Nous ! J'ai moi-même la nationalité belge, je l'ai choisie à l'âge de dix-huit ans. Auparavant, j'étais apatride. Mon père l'est resté car, lui, il devait payer pour devenir Belge. Il avait le choix entre la petite naturalisation, qui coûtait moins cher et ne permettait pas de voter, et la grande naturalisation, plus chère mais dans laquelle était comprise le droit de vote.

K. V. : Et le poète Puškin, a-t-il eu des contacts avec la Belgique?

A. P. :Non. Peut-être en a-t-il eu dans le cadre de son premier emploi au Ministère des Affaires étrangères... Nous l'ignorons. Sinon, ses contacts avec les pays d'Europe étaient avant tout littéraires. Or, au début du XIX ${ }^{\mathrm{e}}$ siècle, je ne pense pas qu'il y avait de grands écrivains en Belgique, ils sont venus plus tard.

E. M. : Il y a une rumeur qui court selon laquelle le manuscrit du journal intime de Puškin se trouverait en Belgique...

A. P. :C'est une rumeur, c'est faux. Ce soi-disant journal intime, qui ressemble plus à un livre pornographique, n'a même pas été écrit par Puškin. Une personne aurait soidisant trouvé ce manuscrit et l'a attribué au poète ${ }^{4}$, mais les historiens ont démenti son lien avec Puškin. Nous avons voulu l'attaquer en justice, mais on nous a déconseillé de le faire car cela lui aurait fait de la publicité pour la vente de son livre, qui a d'ailleurs été traduit dans plusieurs langues.

Nous sommes tout à fait impuissants face à cela. De la même manière qu'avec Kenneth Puškin qui se fait passer pour un descendant du poète alors qu'il n'a aucun lien avec lui. Les directeurs des musées Puškin le laissent faire car il leur donne de l'argent pour assurer le bon fonctionnement des musées ou pour des publications. Il a aussi ouvert un musée Puškin au Mexique. Mais quand on l'interviewe, il ne parvient manifestement pas à expliquer sa généalogie.

K. V. : Vos aïeuls ont-ils, eux aussi, vécu avec une forte conscience d'être descendants du poète ? Ont-ils fait des actions en ce sens ?

A. P. :Du point de vue historique, ils en ont toujours été conscients. Par contre, nul n'a rien accompli pour cela. Nous sommes donc les seuls. En effet, nous avons créé cette Fondation pour mieux faire connaître Puškin en Belgique.

E. M. : Entretenez-vous des contacts avec les «pouchkinologues»?

A. P. :Oui, avec Viktor Rusakov, un grand chercheur de Pskov. C'est notamment avec lui que j'ai parlé du cas de Kenneth Puškin. Il a d'ailleurs écrit un livre à ce sujet ${ }^{5}$. 


\section{K. V. : Parmi les descendants de Puškin, y a-t-il des poètes?}

A. P. :Non. Personnellement, je n'écris pas ou du moins seulement à titre privé. De plus, une légende en Russie dit que Puškin aurait interdit à ses descendants d'écrire. Cela m'arrange bien.

M. P. : Toutefois, il y a un descendant en Amérique qui écrit. Il a présenté son livre lors de la grande réunion des Puškin à Moscou. Bien qu'il ne portât pas le nom du poète, il a pris le nom de sa grand-mère, changeant son nom de « Adam » en " Puškin ». D'ailleurs, nombreux sont ceux à l'avoir fait parmi les descendants. Parce que cela fait bien.

E. M. : Je souhaiterais aborder encore une problématique. Ma question sera peut-être rhétorique, mais pensez-vous que Puškin est traduisible en français ?

A. P. :C'est effectivement difficile, on ne parvient pas à reproduire la profondeur de la langue. C'est plus facile en anglais, en allemand.

A. P.:Je terminerai par une chose intéressante que pratiquement nul ne sait: nous sommes liés par mariage à la Maison de Belgique. Au-dessus de la reine Astrid, il y a le roi Oscar qui a une fille prénommée Sophie et un fils prénommé Nicolas. C'est ce dernier qui a épousé la fille de Puškin.

M. P. :Et moi, j'ai du sang de la famille de Nikolaj Gogol dans les veines puisque ma grand-mère a épousé le neveu de l'écrivain.

Pour de plus amples informations sur les activités de la Fondation A.S. Pouchkine : http:// www.pouchkine.org.

\section{NOTES}

1. Wim Coudenys a transmis cette information aux Puškin de vive voix, lors d'une visite chez eux.

2. Coudenys W., Leven voor de tsaar. Russische ballingen, samenzweerders en collaborateurs in België (Vivre pour le tsar. Les exilés russes, conspirateurs et collaborateurs en Belgique), Davidsfonds, Leuven, 2004, $365 \mathrm{p}$.

3. Vladimir Ronin est docteur en slavistique et spécialiste de l'histoire de l'émigration russe en Belgique. Son dernier livre s'appelle «Russkoe Kongo » : 1870-1970 (« Le Congo Russe » : 1870-1970), Russkij put', Moskva, 2009, 520 p.

4. Pushkin A. S., Secret Journal 1836-1837 [traduit du russe et publié par Mikhail Armalinskij], MIP Co, Minneapolis, 1986, $91 \mathrm{p}$.

5. Rusakov V., o lžepotomkakh A. S. Puškina. I ne toL'ko o nikh (Les faux descendants de Puškin. Et pas seulement eux), B/i, Pskov, 2002, 134 p. 
INDEX

Index géographique : Belgique, Russie

Mots-clés : littérature russe

Index chronologique : romantisme, XIXe siècle

\section{AUTEURS}

\section{ERIC METZ}

Université de Gand (Belgique), Haute Ecole Artesis (Anvers, Belgique) ; membre du comité de rédaction de « Slavica Bruxellensia »

\section{KATIA VANDENBORRE}

Doctorante à l'Université Libre de Bruxelles (Belgique) et à l'Université de Varsovie (Pologne) ; membre du comité de rédaction de "Slavica Bruxellensia » 